\title{
Angioplastia cirúrgica de óstio e tronco coronariano: experiência de oito casos
}

Danton R. R. LOURES*, Edison J. RIBEIRO*, Rui Sequeira de ALMEIDA*, Maria Joăo A. FERREIRA*, Ronaldo R. L. BUENO*, Paulo Mauricio P. ANDRADE*, Marcos Augusto A. PEREIRA*, Paulo Roberto F. ROSSI*.

LOURES, D. R. R.; RIBEIRO, E. J.; ALMEIDA, R. S.; FERREIRA, M. J. A.; BUENO, R. R. L.; ANDRADE, P. M. P.; PEREIRA, M. A. A.; ROSSI, P. R. F. - Angioplastia cirúrgica de óstio e tronco coronariano: experiência de oito casos. Rev. Bras. Cir. Cardiovasc., 5(3) 154-159, 1990.

RESUMO - No período de abril de 1980 a março de 1990, foram realizadas oito cirurgias de angioplastia de óstio e/ou tronco de coronária esquerda ou direita. Não houve mortalidade imediata e esses pacientes foram acompanhados por um período de 1-109 meses (43,2 meses). Houve melhora clínica e de classe funcional. Seis pacientes fizeram estudo cineangiográfico das coronárias e do ventrículo esquerdo, revelando uma anatomia de óstio e tronco adequada na área operada, e melhora da contratilidade do ventrículo esquerdo. Na evolução tardia houve dois óbitos. Um paciente morreu a 39 meses do pósoperatório, com insuficiência cardíaca congestiva e dor anginosa; o outro faleceu em acidente rodoviário, após 109 meses da cirurgia. Estes resultados permitem concluir que a angioplastia por lesōes obstrutivas em óstio ou tronco coronário direito ou esquerdo, isoladas ou associadas a outros defeitos, é um procedimento cirúrgico com baixo risco imediato, com evolução favorável a longo prazo e que pode ser considerado como tratamento opcional para revascularizaçāo coronária.

DESCRITORES: miocárdio, revascularização, cirurgia.

\section{INTRODUÇĀO}

A incidência de lesão de tronco de coronária esquerda (TCE) é ao redor de $13 \%{ }^{19}$ e pode vir sem qualquer envolvimento da árvore coronária ${ }^{4,22}$. 0 comprometimento de $50 \%$ ou mais do TCE é considerado como lesāo de alto risco ${ }^{4}$. O tratamento clínico da lesão do TCE revelou índice de sobrevida em quatro e seis anos, respectivamente, de 65 a $44 \%{ }^{4}$. A revascularização direta do miocárdio temse tornado o procedimento clássico para pacientes com doença obstrutiva da artéria coronária ${ }^{14}$. A veia safena e a artéria mamária têm sido, ambas, empregadas para este tipo de abordagem. A endarterectomia dos óstios coronários em caso de lues 2. 3, 6.8, 15, 18 e a angioplastia em casos de aterosclerose e lesōes iatrogênicas foi realizada, com resultados

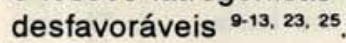

HITCHCOCK et alii ${ }^{16.17}$ apresentaram, em 1983, a primeira série de nove casos de angioplastia de óstio de $C E$, com resultados positivos. No Brasil, CARVALHO et alii ${ }^{7}$ apresentaram em 1984, o primeiro caso com sucesso publicado de angioplastia de óstio de coronária esquerda. O presente trabalho tem como objetivo analisar uma experiência de oito casos de angioplastia cirúrgica de óstio e tronco coronário, observando os seus resultados cirúrgicos imediato e tardio.

\section{CASUÍSTICA E MÉTODOS}

No período de abril de 1980 a março de 1990 , foram operados oito pacientes com lesão de óstio e/ou tronco de coronária esquerda ou direita. A

Trabalho realizado no Serviço de Cirurgia Cardiovascular do Hospital Evangélico de Curitiba. Curitiba, PR. Brasil.

Apresentado ao $17^{\circ}$ Congresso Nacional de Cirurgia Cardiaca. Belo Horizonte, MG, 6 e 7 de abril, 1990.

* Do Serviço de Cirurgia Cardiovascular do Hospital Evangélico de Curitiba e Fundação Cardíaco - F.A.P.E.C.

Endereço para separatas: Danton R. R. Loures. Rua Sảo Pedro. 122. Apto. 2001, $20^{\circ}$ andar. 80035 Cabral. Curitiba, PR, Brasil. 
LOURES, D. R. R.; RIBEIRO, E. J.; ALMEIDA, R. S.; FERREIRA, M. J. A.; BUENO, R. R. L.; ANDRADE, P. M. P.; PEREIRA, M. A. A.; ROSSI, P. R. F. - Angioplastia cirúrgica de óstio e tronco coronariano: experiência de oito casos. Rev. Bras. Cir. Cardiovasc., 5(3): 154-159, 1990.

idade variou de 44 a 61 anos, com média de 53,8 anos. Nāo houve predomínio de sexo neste grupo. Nos períodos pré-operatório e pós-operatório, foram analisados: quadro clínico, classificação funcional pela angina, achados das lesōes coronárias, função do VE no estudo hemodinâmico, complicaçăo e mortalidade.

A abordagem cirúrgica da angioplastia se fez através de toracotomia mediana transesternal, canulaçāo arterial via aorta e canulaçāo venosa única via átrio direito. Foi empregado oxigenador de bolhas Macchi. Para controle da funçāo cardíaca, foram instalados pressāo arterial média, pressão venosa central, pressāo de átrio esquerdo, marcapasso externo e monitoragem cardíaca. A drenagem torácica única e o fechamento do tórax se fizeram por planos anatômicos. A proteção miocárdica foi realizada com solução cardioplégica cristalóide gelada a $4^{\circ}$ centígrados, única, em raíz de aorta ou em óstios coronários, soro gelado no saco pericárdico cada $10-15^{\prime}$ e hipotermia moderada sistêmica através da circulação estracorpórea (CEC) entre 26 e $28^{\circ} \mathrm{C}$.

Para o tratamento das lesōes da coronoária esquerda quando localizadas isoladamente no óstio, a abordagem cirúrgica se fez por uma incisāo látero-posterior direita na aorta ascendente, estendedo-se até próximo à bifurcaçāo dos ramos descendente anterior e cincunflexo. A ampliaçāo com um segmento de pericárdio bovino abrangia tronco, óstio e aproximadamente $3 \mathrm{~cm}$ da aorta ascendente (Figura 1).

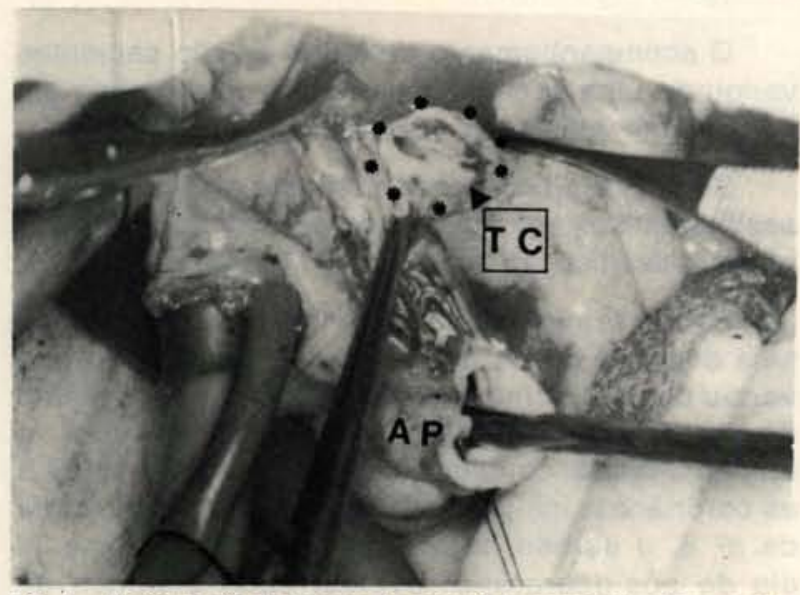

Fig. 1 - Angioplastia de tronco da coronária esquerda. Transecçâo supravalvar da artéria pulmonar (AP), dissecçảo e incisảo longitudial do tronco da coronária esquerda (T. C.)

Quando as lesōes obstrutivas se localizavam no tronco da coronária esquerda, a abordagem se fez por uma incisāo ântero-lateral esquerda estendendo-se para óstio, tronco até imediatamente antes da sua bifurcaçāo. A transecçāo da artéria pulmonar foi a opçāo para melhor exposiçāo da anatomia (Figura 2).

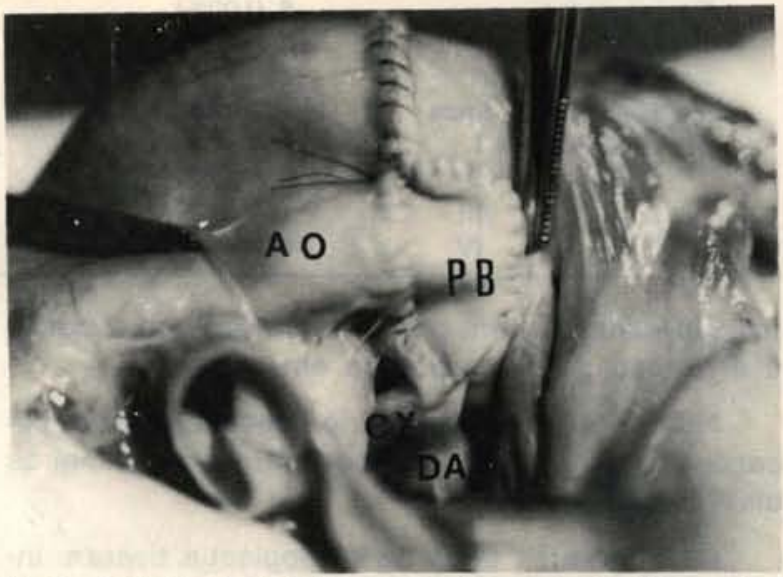

Fig. 2 - Angioplastia de óstio de coronária esquerda. Dissecção e incisâo látero-posterior direita da aorta $(\mathrm{Ao})$ estendendo-se até óstio e tronco de coronária esquerda. Ampliação com um retalho de pericárdio bovino (PB).

Cx: artéria circunflexa; DA: artéria descendente anterior.

Quando as lesōes comprometiam óstio e/ou tronco da coronária direita logo após a sua origem, a incisăo se iniciou na coronária direita, óstio até $15 \mathrm{~mm}$ da parede da aorta ascendete (Figura 3 ).

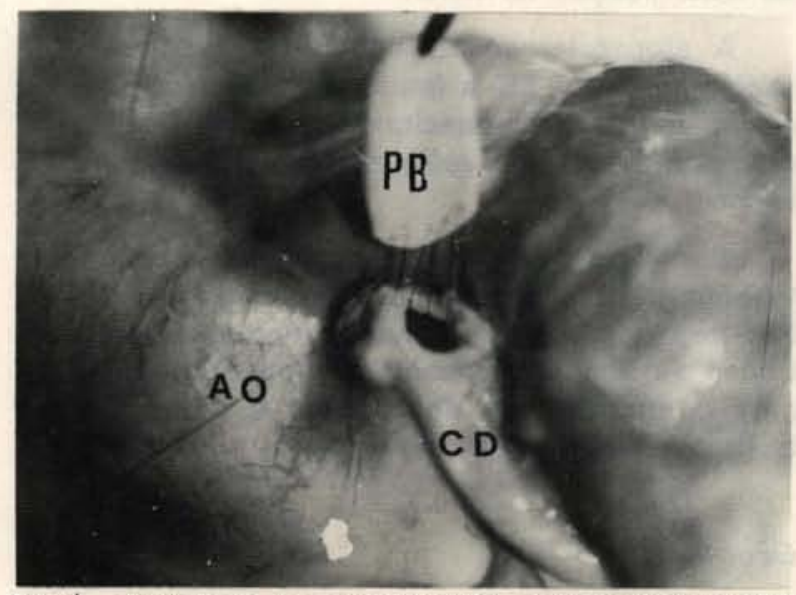

Fig. 3 - Angioplastia de coronária direita. Incisảo longitudinal da coronária direita estendendo-se até óstio e $15 \mathrm{~mm}$ da parede anterior da aorta ascendente e ampliação com retalho de pericárdio bovino. $A o=$ aorta: $\mathrm{CD}=$ coronária direita $; \mathrm{PB}=$ pericárdio bovino.

Os materiais empregados para a ampliação das artérias e óstios coronários foram: veia sefena e retalho de pericárdio bovino.

Esta técnica esteve associada a revascula- 
LOURES, D. R. R.; RIBEIRO, E. J.; ALMEIDA, R. S.; FERREIRA, M. J. A.; BUENO, R. R. L.; ANDRADE, P. M. P.; PEREIRA, M. A. A.; ROSSI, P. R. F. - Angioplastia cirúrgica de óstio e tronco coronariano: experiência de oito casos. Rev. Bras. Cir. Cardiovasc., 5(3): 154-159, 1990.

TABELA 1

\begin{tabular}{|c|c|c|c|}
\hline QUADRO CLÍNICO & PRÉ & PÓS-IMEDIATO (30 dias) & PÓS-TARDIO (1-109 meses) \\
\hline Assintomático & - & - & $6(75,0 \%)$ \\
\hline Dor anginosa & $8(100 \%)$ & - & - \\
\hline Dispnéia e cansaço & $3(37,5 \%)$ & - & $1(12,5 \%)$ \\
\hline Arritmia transitória & - & $5(62,5 \%)$ & - \\
\hline Insuficiência cardíaca & $1(12,5 \%)$ & - & $1(12,5 \%)$ \\
\hline Classe funcional I & - & $7(87,5 \%)$ & $6(75,0 \%)$ \\
\hline II & $1(12,5 \%)$ & $1(12,5 \%)$ & $1(12,5 \%)$ \\
\hline III & $1(12,5 \%)$ & - & $1(12,5 \%)$ \\
\hline IV & $6(75,0 \%)$ & - & - \\
\hline
\end{tabular}

rizaçāo clássica com ponte de safena em 2 casos e a retroca da valva aórtica em um caso.

No período pós-operatório imediato, as complicaçōes mais freqüentes ocorreram nos primeiros dias de UTI (Tabela 1).

Os diferentes tipos de angioplastia tiveram indicaçōes específicas (Tabela 1).

\section{RESULTADOS}

\section{Período Pré-operatório}

A dor anginosa esteve presente no quadro clínico em $100 \%$ dos casos (Tabela 1 ).

Setenta e cinco por cento dos pacientes encontraram-se, no período pré-operatório, com grau IV da classificaçăo funcional.

A aterosclerose foi considerada como fator etiológico em $62,5 \%$ e fatores iatrogênicos em três casos, sendo dois relacionados ao cateterismo e um após a troca da valva aórtica por prótese biológica.

O estudo hemodinâmico revelou comprometimento do óstio de coronária esquerda em $62,5 \%$. Em três dos casos, houve comprometimento do tronco e/ou lesōes associadas (obstruçōes de ramos coronários em dois casos e disfunção de bioprótese aórtica em um caso).

\section{Cirurgia}

Dos tipos de correçōes cirúrgicas, a angioplastia de $C E$ por via posterior foi realizada em $50 \%$ dos casos.

Os procedimentos associados, como revascularização direta com ponte de safena, e troca valvar, ocorreram em três dos casos.

A angioplastia cirúrgica por via anterior foi indicada quando havia lesāo isolada do troco, quan- do havia lesōes associadas de tronco e óstio ou, ainda, quando havia lesōes nos ramos coronários após bifurcação do tronco.

A via posterior foi indicada somente quando havia lesão isolada de óstio. A associação de outras lesōes nos ramos coronários não invalidavam esta abordagem.

A angioplastia cirúrgica para coronária direita teve sua indicaçāo associada a retroca de uma bioprótese de pericárdio bovino.

\section{Pós-operatório Imediato}

No período de pós-operatório imediato, considerado até 30 dias, as arritmias foram as complicaçōes mais freqüentes e observadas principalmente no período de UTI (Tabela 1).

\section{Pós-operatório Tardio}

O acompanhamento de todos os oito pacientes variou de uma semana a dez anos, oferecendo um seguimento médio de 42,7 meses, ou 3,6 anos.

Setenta e cinco por cento deste grupo estavam assintomáticos e o mesmo percentual se encontrava na classificação funcional I (Tabela 1).

Foram reavaliados pelo estudo hemodinâmico seis dos oito casos da série, em um período que variou de uma semana a 56 meses, sendo a média de 15,1 meses. Neste grupo de estudo, a angioplastia foi considerada adequada em $100 \%$ e as coronárias, inalteradas em cinco deles. No caso de $n^{2} 8$, o estudo hemodinâmico realizado no $7^{\circ}$ dia de pós-operatório revelou: óstio e tronco de coronária esquerda livres de lesão e com boa anatomia; bifurcaçăo precoce dos ramos da descendente anterior (DA) e circunflexa (Cx); oclusão total na origem da $\mathrm{Cx}$; ventriculografia esquerda com hipocontratilidade póstero-lateral e ponte de veia safena para DA permeável. A função do ventrículo esquerdo foi considerada normal em $60 \%$ dos casos. 
LOURES, D. R. R.; RIBEIRO, E. J.; ALMEIDA, R. S.; FERREIRA, M. J. A.; BUENO, R. R. L.; ANDRADE, P. M. P.; PEREIRA, M. A. A.; ROSSI, P. R. F. - Angioplastia cirúrgica de óstio e tronco coronariano: experiência de oito casos. Rev. Bras. Cir. Cardiovasc., 5(3): 154-159, 1990.

A mortalidade tardia foi observada em dois pacientes ( $n^{\circ}$ s 1 e 4 ) com 9 anos e 1 mês e 3 anos e 3 meses, respectivamente. O paciente de $n^{2} 1$ morreu em conseqüência de um acidente rodoviário; o de $n^{2} 4$ faleceu de angina e insuficiência cardíaca congestiva.

\section{COMENTÁRIOS}

As lesōes de óstio coronário săo raras, podendo ser adquiridas ou congênitas $e$, quando as localizamos na coronária direita, parecem ser mais freqüentes nas mulheres 21

No nosso grupo nāo houve predomínio de sexo.

O comprometimento do óstio coronário, quando localizado à esquerda, reveste-se da mesma gravidade que a lesão de tronco ${ }^{2,3,6,8}$.

No quadro clínico, a dor anginosa foi a mais freqüente e as suas manifestações foram as mais diversas: síndrome intermediária, angina invalidante, angina de recente começo, etc. Setenta e cinco por cento dos nossos casos encontravam-se na classe funcional IV de angina.

As causas etiológicas das lesōes ostiais săo, basicamente, três, a saber; as decorrentes de estágio avançado da lues ${ }^{3,5,6,13,14,22}$, as decorrentes de aterosclerose ${ }^{2,13,19}$, e as decorrentes de substituiçāo da valva aórtica (iatrogênica) 4, 11, 25. No nosso grupo, a aterosclerose foi a mais observada $(62,5 \%)$.

Os estudos hemodinâmicos têm demonstrado que um número pequeno de pacientes tem estenose isolada do tronco de coronária, sem qualquer envolvimento da árvore coronária ${ }^{8,9,20}$. Neste grupo de oito pacientes operados, apenas dois $(25 \%)$ tinham lesões obstrutivas em outras artérias, que exigiram revascularização associada.

HITCHCOCK et alii ${ }^{16,17}$ apresentaram, em 1982 e 1983, a primeira série de nove casos de angioplastia de óstio de coronária esquerda, com resultados favoráveis, e recomendaram, como abordagem cirúrgica de escolha, a via posterior de aorta áscedente.

A angioplastia por via anterior foi empregada em dois de nossos casos, e tiveram sua aplicaçāo principal nas lesōes isoladas de tronco de coronária esquerda, podendo, ou nāo, transeccionar a artéria pulmonar para melhor exposiçāo anatômica. Quando houve comprometimento isolado de óstio de C E, a via posterior foi opçāo considerada melhor.

As lesōes de óstio de coronária direita săo raras e a possibilidade de origem congênita tem sido considerada ${ }^{21}$.
Em nossa experiência, foram operados dois casos com lesōes em C D, onde as causas atribuídas foram: aterosclerose e iatrogênese na substituição da valva aórtica por bioprótese.

A indicação de angioplastia para C D é menos comum, devido à menor repercussāo clínica que essa artéria oferece. No entretanto, quando está associada a lesōes obstrutivas na C E ou, ainda, quando é francamente dominante, essa abordagem pode ser uma opçăo.

Deve-se observar, durante a dissecção e a incisāo da coronária direita, nāo comprometer os ramos infundibular e do nódulo sinusal, os quais nascem $1 / 3$ inicial 1 .

SULLIVAN \& MURPHY ${ }^{23}$ mencionam o sangramento como uma possível complicação nos atos operatório e pós-operatório imediato, e recomendam abandonar a angioplastia por via posterior, procurando fazer sempre por via anterior. Nāo observamos esse tipo de complicação, apesar de, na metade do número dos nossos casos, a via posterior ter sido empregada. No pós-operatório imediato (30 dias), as alteraçōes do ritmo cardíaco e as complicaçōes respiratórias foram observadas e em caráter transitório. Nāo houve mortalidade nesse período.

O acompanhamento clínico tardio variou de um mês a dez anos, com média de 43 meses. Após a angioplastia, apenas $25 \%$ tinham sintomas com grau II e III na classificaçāo funcional por angina.

Os achados da hemodinâmica realizados em seis dos oito pacientes comprovaram ausência de lesāo em óstio ou tronco e melhoria da funçāo do ventrículo esquerdo. No caso de $n^{\circ} 8$, o estudo hemodinâmico de controle após a cirurgia revelou obstruçāo total na origem da artéria circunflexa (Cx). A análise da coronariografia de pré-operatório revelou que a coronária esquerda apresentava um tronco curto e uma origem precoce da artéria $\mathrm{Cx}$. $\mathrm{O}$ fato da $\mathrm{Cx}$ nascer mais próximo ao óstio do que o habitual pode ter favorecido a iatrogênese cirúrgica.

No período de evoluçāo tardia, foram observados dois óbitos: um por atropelamento e outro por insuficiência cardíaca congestiva e angina, respectivamente com nove e três anos da cirurgia. Não houve mortalidade imediata, havendo, por outro lado, apenas um caso de óbito tardio relacionado à cirurgia.

\section{CONCLUSĀO}

A angioplastia para lesōes obstrutivas de óstio e/ou de tronco de coronária esqueda e/ou direita, isoladas ou associadas com outros defeitos, pode ser considerada como procedimento cirúrgico opcional, de baixo risco e com evoluçāo tardia favorável. 
LOURES, D. R. R.; RIBEIRO, E. J.; ALMEIDA, R. S.; FERREIRA, M. J. A.; BUENO, R. R. L.; ANDRADE, P. M. P.; PEREIRA, M. A. A.; ROSSI, P. R. F. - Angioplastia cirúrgica de óstio e tronco coronariano: experiência de oito casos. Rev. Bras. Cir. Cardiovasc., 5(3): 154-159, 1990.

\section{RBCCV 44205-119}

LOURES, D. R. R.; RIBEIRO, E. J.; ALMEIDA, R. S.; FERREIRA, M. J. A.; BUENO, R. R. L.; ANDRADE, P. M.; PEREIRA, M. A. A.; ROSSI, P. R. F. - Ostium and left coronary angioplasty: experience with eight cases. Rev. Bras. Cir. Cardiovasc., 5(3): 154-159, 1990.

ABSTRACT: Between April 1980 and March 1990 we performed eight surgical angioplasties of the left main coronary artery and/or its ostium and of the right coronary artery's ostium. There was no early mortality, and these patients were followed by a period of one to 109 months (medium of 43.2 months). We have observed improvement in the clinical symptoms and functional class. A new angiocardiography was performed in six patients, for angioplasty control and left ventricular ( $L$ V) function evaluation if there was an adequate anatomy on the surgical corrected site and an improvement on the $L V$ contractility and ejection fraction. There were two late deaths. One patient died 39 of postoperative months in congestive heart failure and with angor pectoris, the other one in a car accident 109 months after the surgical correction. These results lead us to conclude that surgical angioplasty for ostium, right or left main coronary artery due to obstructive lesions, isolated or associated with other defects, is an optimal procedure to coronary revascularization.

DESCRIPTORS: myocardial revascularization, surgery.

\section{REFERÊNCIAS BIBLIOGRÁFICAS}

ANDERSON, R. H.; BECKER, A. E.; KIRKLIN, J. W. Atlas fotográfico de anatomia cardíaca. São Paulo, Livraria Santos Editora Ltda., 1983. p. 6.5 e 6.6.

BARNER, H. B.; CODD, J. E.; MUDD, J. G.; KAISER, G. C.; TYRAS, D. H.; LAKS, H.; WILLMAN, V. L. Nonsyphilitic coronary ostial stenosis. Arch. Surg. 112: 1462-1466, 1977.

BECK, W; BERNARD, C. N.; SCHRIRE, V. - Syphilitic obstruction of coronary ostia successfully treated by endarterectomy. Br. Heart J., 27: 911-915, 1965.

BJORK, V. O.; HENZE, A.; SZAMOSI, A. - Coronary ostial stenosis: a complication of aortic valve replacement or coronary perfusion? Scand. J. Thorac. Cardiovasc. Surg., 10: 1-6, 1976.

5 BLONDEAU, P. \& DUBOST, C. - Cure chirurgicale sous hypothermie profonde de la coronarite ostiale syphilitique: a propos de 2 cas operés avec succés. Ann. Chir. Thorac. Cardiovasc., 1: 802 806, 1962 ,

CARNEIRO, R. C.; LION, M. F.; OLIVEIRA, P. R. G.; SAN JUAN, E. - Coronarite ostial sifilítica. Arq. Bras. Cardiol., 29: 235-239, 1976.

7 CARVALHO, R. G.; RIBEIRO, E. J.; BROFMAN, P. R.; LOURES, D. R. R. - Angioplastia cirúrgica do óstio da coronária esquerda: relato de caso Arq. Bras. Cardiol., 42, 335-360, 1984.

COHN, L. H. - Coronary ostial stenosis. Circulation, 50: $413-414,1974$.

COHN, L. H. \& COLLINS, J. - Reduced mortality following revascularization surgery for left main coronary stenosis. In: Coronary artery medicine and surgery: concepts and controversies. New York, Appleton, 1975. p. 756.
10 CONNOLLY, J. E.; ELDRIDGE, F. L.; CALVIN, J. W.; STEMMER, E. A. - Proximal coronary-artery obstruction: its ethiology and treatment by transaortic endarterectomy. N. Engl. J. Med., 271: 213-219, 1964.

11 CREXELLS, C.; CARALPS, J. M; ORIOL, A. - Coronary angioplasty in iatrogenic coronary artery stenosis. J. Thorac. Cardiovasc. Surg., 85: 634-637, 1983.

12 D'ALLAINES, C.; LACOUR-GAYET, BLONDEAU, $P$.; PIWNICA, A.; CARPENTIER, A.; SELIEUR, P.; DUBOST, C. - Résultats à distance des coronarité ostiales opérées. Arch. Mal.Coeur, 11: 1173-1179, 1979.

13 EFFLER, D. B.; SONES, F. M.; FAVALORO, R. G.; GROVES, L. K. - Coronary endarterectomy with patch reconstruction: clinical experience with 34 cases. Ann. Surg., 162: 590-594, 1965.

14 FAVALORO, R. G. - Direct myocardial revascularization. A 10 year journey: myths and realities. Am. J. Cardiol., 43: 109-114, 1979.

15 GERBAUX, A.; ORY, A.; DUBOST, C.; LENEGRE, T. - Un noveau cas d'angine de poitrine due à une coronarité ostiale syphilitique traitée par la désobstruction chirurgicale des orifices coronaires. Arch. Mal. Couer, 55: $721-727,1962$.

16 HITCHCOCK, J. F. \& ROBLES DE MEDINA, E. O. Angioplasty of the left main coronary artery for isolated main coronary artery diasease. Am. J. Cardiol., 49: 956, 1982. (Resumo).

17 HITCHCOCK, J. F.; ROBLES DE MEDINA, E. O.; JAMBROES, G. - Angioplasty of the left main coronary artery disease. J. Thorac. Cardiovas. Surg. 85: 880-884, 1983. 
LOURES, D. R. R.; RIBEIRo, E. J.; ALMEIDA, R. S.; FERREIRA, M. J. A.; BUENO, R. R. L.; ANDRADE, P. M. P.; PEREIRA, M. A. A.; RoSSI, P. R. F. - Angioplastia cirúrgica de óstio e tronco coronariano: experiência de oito casos. Rev. Bras. Cir. Cardiovasc., 5(3): 154-159, 1990.

JEFFERY, D. L.; VIJAYANAGAR, R.; BOGNOLO, D. A.; ECKSTEIN, P. F.; SPOTO Jr., E.; NATARAJAN, P.; WILLIARD, E. H.; CONNAR, R. G. - Surgical treatment of 200 consecutive patients with left main coronary artery disease. Ann. Thorac. Surg., 36: 193-196, 1983.

KIRKLIN, J. W. \& BARRATT-BOYES, B. G. - Cardiologic surgery. New York, Editora John Wiley \& Sons Inc., 1986. 1550 p.

21 LEA, J. W.; PAGE, D. L.; HAMMON Jr., J. W. - Congenital ostial stenosis of the right coronary artery refaired by vein patch angioplasty. J. Thorac. Cardiovasc. Surg., 92: 796-798, 1986.

MICHAUD, P.; TERMET, H.; CHASSIGNOLLE, T.; DALLOZ, C.; AGÉ, C.; DELAYE, J.; GRÉS, B.; CHAMPSAUR, G. - Coronarité ostiale syphilitique: cinq cas de désobstruction chirurgicale dont deux comportant un remplacement valvulaire pour insuffisance aortique. Arch. Mal. Coeur, 63, 674-693, 1970.

23 PRITCHARD, C. L.; MUDD, J. G.; BARNER, H. B. Coronary ostial stenosis. Circulation, 52: 46-48, 1975.

24 SULLIVAN, J. A. \& MURPHY, D. A. - Surgical repair of stenotic ostial lesions of the left main coronary artery. J. Thorac. Cardiovasc. Surg., 98, 33-36, 1989.

YATES, J. D.; KIRSH, M. M.; SODMEAN, T. M.; WALTON Jr., J. A.; BRYMR, J. F. - Coronary ostial stenosis: a complication of aortic valve replacement. Circulation, 69: $530-534,1974$.

\section{DISCUSSĀO}

\section{DR. JOSÉ CARLOS DE ANDRADE}

São Paulo, SP

O trabalho apresentado pelo Dr. Danton, e que tivemos oportunidade de analisar, é um trabalho bem elaborado, bem redigido, ilustrado, de experiência pessoal e que conclui ser a angioplastia de óstio e tronco um procedimento cirúrgico opcional nas lesōes ostiais. Sua discussão nos pa- rece muito oportuna porque lesōes de óstio não têm tratamento de consenso, podendo ser realizados: 1) revascularização miocárdica (a mais rotineiramente empregada); 2) plastia cirúrgica (como apresentada pelo Dr. Danton); 3) reimplante de óstio para coronária direita; 4) alguns hemodinamicistas mais afoitos já estudam formas de intervenção por cateterismo. A revascularizaçāo miocárdica, evidentemente, nāo é cirurgia definitiva. Todos sabemos da durabilidade de uma ponte de safena praticamente obrigatória nessa opção. Como em $60 \%$ dos casos aqui apresentados a lesão ostial estava isolada, justifica-se, a nosso ver, o tratamento empregado. A plastia cirúrgica, apesar de alguns resultados sofríveis em trabalhos antigos, parece-nos a terapêutica de maior durabilidade. Discutiríamos, isto sim, que material empregar: retalho de veia safena ou de pericárdio? A veia é um enxerto bem testado e aprovado para confecção de pontes, não, porém, para substituir parte da aorta. Achamos válido lembrar a Lei de Laplace, onde a tensāo é diretamente proporcional à pressão e ao raio da cavidade. Apesar da pressão ser a mesma na aorta ou na ponte de safena, o raio da aorta é, muitas vezes, maior que o da veia, de tal modo que a tensão na parede do enxerto usado na plastia de aorta é, muitas vezes, maior que a exercida na parede do enxerto usado como ponte. Assim, apesar de ser a veia um tecido mais delicado e atraente para este tipo de cirurgia, acreditamos ser interessante utilizar tecido mais resistente, como o pericárdio. Entretanto, como a plastia envolve não só a parede aórtica, mas também estrutura mais delicada como a artéria coronariana, nāo será todo e qualquer pericárdio, de qualquer espessura, que terá indicação. Daí a validade de trabalharmos com material industrializado e já com espessura perfeitamente dimensionada. O pericárdio bovino tem sua espessura variável de 0,20 a 0,40 milímetros. O grupo de São José do Rio Preto, e nós mesmos, temos já experiência bem sucedida com uso de pericárdio de 0,25 milímetros para tratamento de aneurismas de aorta. Essa espessura $(0,25)$ está, também, portanto, testada e, sendo pericárdio delgado, nos parece a recomendada para essa plastia. Encerramos reiterando nossos cumprimentos aos autores do trabalho e em especial ao Dr. Danton, pela magnífica apresentação. 